\title{
Does Prigogine's Non-linear Thermodynamics Support Popular Philosophical Discussions of Self-Organization?
}

\author{
Alexander Pechenkin \\ Faculty of Philosophy, \\ Lomonosov Moscow State University, \\ Leninskie Gory, \\ Moscow GSP-1, Russia \\ E-mail: a_pechenk@yahoo.com
}

\begin{abstract}
The article is concerned with the philosophical talks which became popular in the 1980 s and have kept their popularity till now-the philosophical essays about self-organization. The author attempts to find out as to which extent are these essays founded on the scientific theory to which they regularly refer, that is, Ilya Prigogine's non-linear thermodynamics. The author insists that the equivalent of self-organization in Prigogine's theoretical physics is the concept of dissipative structure. The concept of selforganization, as it is used in philosophical literature, presupposes a sequence of extrapolations, the first extrapolation being conducted by Prigogine and his coauthors. They became to use the concept of dissipative structure beyond the rigorous theory of this phenomenon. The subsequent step was that the scientific term "dissipative structure" was replaced by the vague concept "self-organization" in many popular and semi-popular books and papers. The author also emphasizes that by placing the concept of self-organization into the framework of philosophical concepts (the picture of the world, the ideals of scientific thought, the contemporary scientific revolution, etc.) a philosopher conducts the extrapolation of extrapolation and comes to a kind of what Edmund Husserl called Weltanschauung ('worldview') philosophy.
\end{abstract}

Keywords: dissipative structure, extrapolation, non-linear thermodynamics, self-organization, Weltanschauung philosophy 


\section{Introduction}

Philosophical talks about self-organization became an essential component of scientific literature at the end of the $20^{\text {th }}$ century and in the $21^{\text {st }}$ century. To cite Wikipedia, "Self-organization is a process where some form of global order or coordination arises out of the local interactions between the components of an initially disordered system. [...] Self-organization occurs in a variety of physical, chemical, biological, social and cognitive systems." (Wikipedia, n.d.) The most robust and unambiguous examples of self-organizing systems are from the physics of non-equilibrium processes. Self-organization is also relevant to chemistry.

The concept of self-organization is central to the description of biological systems, from the subcellular to the ecosystem level. There are also cited examples of "self-organizing behavior" found in the literature of many other disciplines, both in the natural sciences and the social sciences such as economics or anthropology. [...]

Self-organization as a word and concept was used by those associated with general systems theory in the 1960s, but did not become commonplace in the scientific literature until its adoption by physicists and researchers in the field of complex systems in the 1970s and 1980s. After Ilya Prigogine's 1977 Nobel Prize, the thermodynamic concept of self-organization received some attention of the public, and scientific researchers started to migrate from the cybernetic view to the thermodynamic view. (Wikipedia, n.d.)

An objection is very likely: one can say that Wikipedia does not represent scientific culture. However we can refer to a lot of philosophical and scientific publications in which the term "self-organization" is significantly important and even central (for an analytical review of some of them see Näpinen, 1993; 2004; 2014; also Feistel \& Ebeling, 2011).

In many writings the concept of self-organization is connected with Prigogine's non-linear non-equilibrium thermodynamics (see, for example, the above quotation from Wikipedia). Moreover, this theory is often being represented as the basis, or at least as a justification, of the worldview which uses selforganization as a central notion.

The present article is critical. It challenges the belief that self-organization is the conception of Prigogine's thermodynamics. Indeed, it is not a technical concept 
of a scientific theory. Nevertheless, "self-organization" is present in many books written by Prigogine and his coauthors. What is the reason of such popularity? The conception of dissipative structure, which is the characteristic message of Prigogine's theory (the theory of dissipative structures won Prigogine the Nobel Prize in Chemistry), can be treated as a counterpart of the philosophical (and/or scientifically popular) concept of self-organization. However, there is no direct way from the dissipative structure to self-organization. The article will show that the formulation of the concept of self-organization in Prigogine's and his coauthors' writings results from at least two extrapolations: (1) the extrapolation of the "good" exact conception of dissipative structure, the conception elaborated by a theoretical analysis of some experimental phenomena, over a number of similar physical, chemical, biological phenomena, and (2) the extrapolation of the conception of dissipative structure beyond real and hypothetical dissipative structures, described in Prigogine's nonlinear thermodynamics, the formation of the dissipative structure, taking the vague name "self-organization".

In the following section, the definition of the dissipative structure, contained in Prigogine's works, will be formulated. This definition is the basis of extrapolation of the concept of the dissipative structure over a number of phenomena which could be conventionally embraced by this definition. The third section will consider the structure of Prigogine's non-linear non-equilibrium thermodynamics which forms the context of the concept of dissipative structure. It will be noted that the real definition of the dissipative structure could be given within the framework of the theory and any extrapolation of this concept should be founded by the corresponding development of the theory. The fourth section returns to the concept of dissipative structure. I will concentrate on the dissipative structures to which Prigogine and his coauthor refer as paradigmatic examples within the framework of nonlinear non-equilibrium thermodynamics. The fifth section describes the extrapolation of the concept of dissipative structure over a field of close and similar phenomena. The sixth section is concerned with the concept of self-organization. However, this concept is approached in connection with the concept of dissipative structure, following the next step of the extrapolation of the Prigogine's concept, the extrapolation of which transforms it into a concept of philosophical worldview. The final section is dedicated to a philosophical presumption of such an extrapolation. This is the worldview philosophy (Weltanschaunng Philosophie) in Edmund Husserl's terminology. 


\section{The concept and definition of the dissipative structure}

According to Glansdorff and Prigogine (1971):

From the macroscopic point of view it is necessary to distinguish between two types of structure:

- equilibrium structures;

- dissipative structures.

Equilibrium structures may be formed and maintained through reversible transformations implying no appreciable derivation from equilibrium. A crystal is a typical example of equilibrium structure. Dissipative structures have a quite different status: they are formed and maintained through the effect of exchange of energy and matter in non-equilibrium conditions. The formation of cell patterns at the onset of free convection [...] is a typical example of dissipative structure. (Glansdorff \& Prigogine, 1971, p. xv)

Here Glansdorff and Prigogine write about the Bénard cells. In his Nobel lecture, Prigogine (1977) provided the following description of this phenomenon:

It is remarkable that this new type of behavior appears already in typical situations studied in classical hydrodynamics. The example which was first analyzed from this point of view is the so-called 'Bénard instability'. Consider a horizontal layer of fluid between two infinite parallel planes in a constant gravitational field, and let us maintain the lower boundary at temperature $T_{1}$ and the higher boundary at temperature $T_{2}$ with $T_{1}>T_{2}$. For a sufficiently large value of the "adverse" gradient $\left(T_{1}-T_{2}\right) /\left(T_{1}+T_{2}\right)$, the state of rest becomes unstable and convection starts. The entropy production is then increased as the convection provides a new mechanism of heat transport. Moreover, the state of flow, which appears beyond the instability, is a state of organization as compared to the state of rest. Indeed a macroscopic number of molecules have to move in a coherent fashion over macroscopic times to realize the flow pattern. (Prigogine, 1977)

The term 'entropy production' is present in the above piece. This terminology is typical for the Brussels school to which Prigogine belonged. The change of entropy can be split into two parts: the entropy production due to changes inside the system, and the flow of entropy due to interaction with the outside world. According to the second law of thermodynamics the entropy production is never negative. 
The book by Nicolis and Prigogine (1977) offers a slightly different definition, one that refers to the theoretical concept of the thermodynamic branch.

A chemically reacting mixture is described by nonlinear equations having, in general, more than one solution (even when boundary and initial conditions are taken into account). Let us consider the solution corresponding to equilibrium conditions (this implies maximum entropy for isolated systems, minimum Helmholtz free energy for systems at given temperature and volume). We call this solution 'thermodynamic branch.' Suppose we now vary the constraints so as to force the system further and further away from equilibrium. Then, nonequilibrium thermodynamics leads us to formulate condition for the stability of the thermodynamic branch. If this condition is not satisfied the thermodynamic branch may become unstable, and the system may evolve toward a new structure involving coherent behavior. (Nicolis \& Prigogine, 1977, pp. 3-4)

"The new structures," Nicolis and Prigogine write further,

are radically different from the 'equilibrium structures' studied in classical thermodynamics, such as crystals and liquids. They can be maintained in far-from-equilibrium conditions only through a sufficient flow of energy and matter. An appropriate illustration would be a town that can only survive as long as it is a center of inflow of food, fuel, and other commodities and sends out products and wastes. (Nicolis \& Prigogine, 1977, p. 4)

Let us emphasize that here a "town" is a comparison, an illustration, rather than an example of the dissipative structure. True, in many other writings, Prigogine and his coauthors have neglected the difference between an example and an illustration.

In spite of the elements of the theory included in the above definitions, we need to treat them as descriptive definitions (or definitions by description) which allow us to distinguish this phenomenon from other similar phenomena (to some extent like Lewis' definition "man is the animal that laughs") (Robinson, 1954 , p. 104). Such a technique does not allow us to ensure that the observed phenomenon is really a dissipative structure in the technical sense of the word. Moreover, such a definition leads us to use literary examples, removed from science (say, by mentioning the dissipative structure one can refer to the earth as a whole, the earth as an open system subject to the constant flow of energy from the sun). To formulate a real definition of the dissipative structure means to 
develop a theory of this phenomenon (or to construct its theoretical explanation developing already built theories). Here John Locke's example is instructive: the real definition of gold describes the constitution of the insensible parts of the body on which the qualities (mentioned in the nominal definition) depend.

The theoretical explanation of the dissipative structures is provided in Prigogine's and his coauthors' books on nonlinear thermodynamics.

\section{The structure of Prigogine's nonlinear thermodynamics}

In many books on thermodynamics, non-equilibrium phenomena are treated as special problems. Thermodynamics is presented as a linear theory based on a number of well-known postulates. Non-equilibrium nonlinear problems are treated after the main text by inviting some additional assumptions. "Statistical method allows us to solve some problems which cannot be solved within the framework of thermodynamics (for example, to solve the problem of heat capacity)" (Rumer \& Ryvkin, 2008, p. 10).

Prigogine's and his coauthors' books have another structure. They represent "a unified treatment of macroscopic physics, involving both reversible and irreversible processes in both the near and far from equilibrium situations" (Glansdorff \& Prigogine, 1971, p. xvii).

This does not mean that their structure is close to the axiomatic one (or that Prigogine and his coauthors have tried to present their theory axiomatically). Their books (this article mainly refers to Glansdorff \& Prigogine, 1971) are constructed according to the principle of generalizations. It has three levels of generality: regular thermodynamics of the reversible processes, linear thermodynamics of the irreversible process, and nonlinear thermodynamics of the irreversible processes. Within the framework of the third level, the concept of the dissipative structure is constructed and explained.

This three-level structure is evident in the book by Glansdorff and Prigogine (1971). Besides, it is specially described in the article by Nicolis (1970).

It should be noted that the second and third levels result not only from generalization, they also presuppose a specialization which leads to restrictions of generality: each level is connected with additional hypotheses, which request confirmation. As a result, the dissipative structures described and explained in 
Prigogine's and his coauthors' works are not only illustrations and confirmations, but they are caused by the structure of the theory presented in these books.

It can be formulated in another way. Prigogine and his coauthors developed a new conceptual structure of thermodynamics-the dissipative structures presented within the framework of their nonlinear thermodynamics are not only observable phenomena, but they are theoretically loaded by the new conceptual framework.

The first (bottom) level consists in ordinary thermodynamics of equilibrium processes. True, this thermodynamics is enriched by the concepts proper to the Brussels school, which was formed around Théophile de Donder, whose student Prigogine was. The concepts of entropy production and entropy flow were already mentioned above. These concepts are formulated on the first level of thermodynamics. To obtain explicit expressions for them, the concepts of thermodynamic forces and thermodynamic flows are formulated (de Donder formulated the concept of chemical affinity, which is the force that drives chemical reactions, and the corresponding flow is the rate of chemical reaction). Another conventional example of the thermodynamic force is the temperature gradient (a difference in temperature between adjacent parts of a system), a corresponding thermodynamic flow is the irreversible flow of heat.

The second level is linear thermodynamics of irreversible processes. It is based on linear relations between thermodynamic forces and flows (for example, $\left.J_{\alpha}=\sum_{\beta} L_{\alpha \beta} X_{\beta}\right)$. It should be emphasized that these relations are not contained in the apparatus of thermodynamics, but they are additionally constructed and tested. According to Glansdorff and Prigogine (1971, p. 31), the "existence of such phenomenological relations has to be understood as an extra-thermodynamic hypothesis". The paradigm examples of such relations are Fourier's law for heat flow and Fick's law for diffusion. Fourier's law states that the heat flow is proportional to the temperature gradient, Fick's law says that the flow of diffusion (diffusion current) is proportional to the gradient of concentration.

In this connection, the Onsager celebrated reciprocity relations are formulated as $L_{\alpha \beta}=L_{\beta \alpha^{\circ}}$. This can be understood as follows: when the flow $J_{\alpha}$, corresponding to the irreversible process $\alpha$ is influenced by the force $X_{\beta}$ of the irreversible process $\beta$, then the flow $J_{\beta}$ is also influenced by the force $X_{\alpha}$, through the same coefficient $L_{\alpha \beta}$. 
Prigogine and his coauthors emphasized that the Onsager relations did not result from a deductive development of the theory. The Onsager relations have received reliable empirical confirmation.

As was said above, linear thermodynamics of irreversible processes is not only a generalization of ordinary reversible thermodynamics. In order to operate with entropy outside the state of equilibrium, one needs to use the approximation of local equilibrium. This means that within each small mass element of medium there exists a state of local equilibrium for which the local entropy is the same function of the local macroscopic variables as at the state of equilibrium.

As a simple example, expansion of a gas in a pipe corresponds to nonhomogeneous state, as well as to non-equilibrium process. However, at each point the relation between temperature and pressure is still expressed by the same law, e.g. $p v=R T$, as for equilibrium. Likewise, entropy remains defined by Gibbs' formula (here the following relation is meant: $T d s=d E+p d V-\mu_{i} d m_{i}$, where $T$ is absolute temperature, $s$ is entropy, $E$ is internal energy, $p$ is pressure, $\mu_{i}$ is chemical potential per unit mass of constituent $x_{i}, m_{i}$ is mass of the constituent $x_{i}$ ). (Glansdorff \& Prigogine, 1971, pp. 14-15)

In the abovementioned article about the structure of Prigogine's thermodynamics, Nicolis (1970) points to another aspect of the second level (of the linear thermodynamics of irreversible processes):

The local formulation of irreversible thermodynamics has been developed in yet another direction - the search for variational principles. The question one asks is whether there exists a general principle-other than the second law-characterizing non-equilibrium states themselves independently of the details of phenomena occurring in the system. In order to formulate this question quantitatively it is necessary to analyze in some detail the character of a non-equilibrium state in thermodynamics. In an isolated system, one has $d_{e} S=O$ and the second principle implies that entropy increases until it reaches its maximum value. The system thus tends more or less rapidly to a uniquely determined permanent state which is the state of thermodynamic equilibrium. Consider now instead of an isolated system a closed system which can exchange energy with the external world, or an open system which can exchange both energy and matter. In this case, and provided the external reservoirs are sufficiently large to remain in a time independent state, the system may tend to a permanent régime other than the equilibrium 
one. This will be a steady non-equilibrium state. Now this régime is no longer characterized by a maximum of entropy or by a minimum of free energy. In other terms, the variational principles valid in thermal equilibrium cannot be extended beyond this state. It is therefore necessary to look for new principles which generalize the concept of a thermodynamic potential to steady (or slowly varying in time) non-equilibrium states. To this end we subdivide the domain of non-equilibrium phenomena into two parts: the region close to equilibrium and the region of states arbitrarily far from equilibrium. (Nicolis, 1970)

Prigogine has shown that steady states close to equilibrium are characterized by an entirely different variational principle according to which, at the steady state, the entropy production per unit time is a minimum $\frac{d_{i} S}{d t}=\min$ (it is called the principle of the minimum entropy production). It is important to realize that it provides a general evolution criterion. Indeed, the validity of the theorem of minimum entropy production together with the second law implies that a physical system will necessarily evolve to the steady non-equilibrium state and that the latter corresponds to a stable situation. It can be also shown that, under certain conditions, the steady state which, according to the theorem, is characterized by a minimum of dissipation or, so to say, by a maximum of possible stability, is also characterized by a lower value of entropy than at equilibrium: it is a less probable state than the equilibrium.

The third level is nonlinear thermodynamics of irreversible processes. Here Glansdorff and Prigogine put forward what they called the general evolution criterion. Under the assumption of the local equilibrium they demonstrated an inequality which is valid for all macroscopic physics. As is well known, the second law of thermodynamics provides the criterion describing the evolution towards equilibrium. Let us consider a system with fixed boundary conditions incompatible with equilibrium. The Glansdorff-Prigogine evolution criterion (which is reduced to the theorem of minimum entropy production in the linear range) can be presented by the formula $\frac{d_{X} P}{d t} \leq 0$ which says that the change of the forces $X_{\alpha}$ proceeds always in a way as to lower the value of the entropy production. This criterion is independent of any assumption about the phenomenological relations between the flows and the forces.

This was the evolution criterion for dissipative systems. Glansdorff and Prigogine (1971) extend it to include convection processes presupposing collective movement of aggregate of molecules within fluids. Their conclusion 
is the following: "with the exception of non-conservative systems, the evolution criterion is always associated to the entropy production" (Glansdorff \& Prigogine, 1971, p. 124).

The theory of stability presented in Glansdorff's and Prigogine's book is rather complicated. This theory is based on Lyapunov's mathematical theory of stability and Einstein's approach to fluctuations. In his semi-popular book, Prigogine (1980) explains the theory of stability in non-linear thermodynamics by referring to a generalization of the classical thermodynamic theory of stability. If we perturb a system that is near an equilibrium value $S_{e}$, we have

$S=S_{e}+\delta S+\frac{1}{2} \delta^{2} S$

Because the function $S$ is maximum at $S_{e}$, the first-order term vanishes, and therefore the stability is determined by the sign of the second-order term $\delta^{2} S$. When a number of conditions are satisfied, $\delta^{2} S$ is a negative definite quality. Moreover, it can been shown that the time derivative of $\delta^{2} S$ is related to the entropy production, $P$, through the formulae

$$
\frac{1}{2} \frac{\partial}{\partial t} \partial^{2} S=\sum_{\rho} J_{\rho} X_{\rho}=P \geq 0,
$$

where $\delta^{2} S$ is a Lyapunov function and its existence ensures stability (the damping of all fluctuations).

Let us calculate the perturbation for a system in a nonequilibrium state. The time derivative of $\delta^{2} S$ is no longer related to the total entropy production, but to the entropy production arising from perturbation. In other words, we now have another expression for the time derivative of $\delta^{2} S$ :

$$
\frac{1}{2} \frac{\partial}{\partial t} \partial^{2} S=\sum_{\rho} \partial J_{\rho} \partial X_{\rho}
$$

The right hand side is what may be called the excess entropy production at a stationary state. The entropy production contains the thermodynamic forces and flows after the $\sum$ symbol. In turn, the excess entropy production contains the perturbations (disturbances) of the thermodynamic forces and flows after the $\Sigma$ symbol. In other words, this value contains deviations of the forces and flows, their deviations from those values which correspond to the stationary state the stability of which is under consideration.

"There is an essential difference," Prigogine notes, "between the laws for systems at equilibrium and those for systems that are far from equilibrium. The laws of equilibrium are universal. However, far from equilibrium, the behavior may 
become very specific. This is a welcome circumstance because it permits us to introduce a distinction in the behavior of physical systems that would be incomprehensible in an equilibrium" (Prigogine, 1980, p. 93).

"The sign of the excess of entropy production is not prescribed once and for all by the second law of thermodynamics. Therefore we have to discuss its sign according to the phenomenological laws adopted" (Glansdorff \& Prigogine, 1971, p. 75).

Nevertheless, the excess entropy production "seems to be the basic quality whose behavior characterizes the occurrence of new structures and their stability" (Glansdorff \& Prigogine, 1971, p. xxi).

\section{The dissipative structures within the framework of nonlinear thermodynamics}

In Glansdorff's and Prigogine's work, two types of the dissipative structures are taken under discussion: the abovementioned Bénard cells and oscillatory chemical reactions. The Bénard cells provide a "simple example" (Glansdorff \& Prigogine, 1971, p. xii). They have been described in the classical works on hydrodynamics (Landau \& Lifshitz, 1988, pp. 311-318). The Glansdorff-Prigogine treatment follows Subrahmanyan Chandrasekhar's (1961, ch. 2) work on hydrodynamics. However, dissipative structures occur only within the framework of nonlinear thermodynamics.

Glansdorff and Prigogine (1971, p. 149) treat the Bénard cells from the perspective of their theory of evolution and as development of their theory of stability. By simplifying their discussion, one comes to the following description:

The manifold of solutions corresponding to the system at rest will be called the thermodynamic branch. At the Bénard point, the thermodynamic branch becomes unstable. We then have a transition to a new branch.

This transition involves the appearance of a dissipative structure. Indeed, at the critical point the system uses part of its thermal energy to build up the kinetic energy necessary to maintain the macroscopic stationary cellular motion which occurs at the onset of free convection. Then the layer appears as formed by juxtaposed calls aligned to form a regular hexagonal pattern in the horizontal plane. (Glansdorff \& Prigogine, 1971, p. 154) 
Glansdorff and Prigogine (1971, p. 166) also demonstrate that in the Bénard problem, "convection sets in as stationary motion, or in other words, the so called principle of the exchange of stabilities (Chandrasekhar's terminology) is satisfied”.

This description follows the discussion (see Glansdorff \& Prigogine, ch. 11, as here I discuss only the beginning points): (1) consider a horizontal layer of fluid between two infinite parallel planes, in a constant gravitational field, and let us maintain the lower boundary at temperature $T_{1}$, and the higher boundary at $T_{2}$ with $T_{1}>T_{2}$; (2) if the layer is thin, we may neglect the pressure dependence of the density, and the equation of state may be written in the linear form; 3 ) the usual phenomenological laws give us the relations connecting the thermodynamical forces and flows-Fourier's law and the linear laws of Newtonian fluid; (4) apply separately the thermodynamic and hydrodynamic stability conditions (the former condition takes the dissipative processes in liquid under consideration, the latter takes the variation of its kinetic energy under consideration).

This algorithm can be treated as a real definition of the Bénard cells as a dissipative structure.

The other example of the dissipative structures in the Glansdorff-Prigogine's book refers to chemical kinetics. However, this illustration turned out to be a difficult problem. If the description of the Bernard problem proceeded from the theory "presented in the previous chapters", chemical kinetics requested additional theoretical constructions.

According to Glansdorff and Prigogine:

Both in hydrodynamics and in chemical kinetics instabilities due to nonlinear effects may occur far from equilibrium. In hydrodynamics, non-linear effects are generated by the inertial terms (critical Reinolds number). However, the chemical kinetic problem may correspond to a practically infinite variety of possible mathematical structures. Indeed in the chemical case we are concerned with an arbitrary number of steps, each of which involves usually a monomolecular or bimolecular mechanism. One of our main aims will be to investigate under what conditions instabilities have to be expected in such schemes. It will appear from this study, that some autocatalytic effect, in general sense of the term, is always required: the same compound has to fulfill at least two different function in the reaction scheme. (Glansdorff \& Prigogine, 1971, p. 82) 
Autocatalysis is usually called the catalysis of the reaction by its products. Glansdorff and Prigogine use the term 'autocatalysis' in a more embracing sense which allows them to extrapolate it over subtle schemes of reactions. They came to an important conclusion which has a character of prediction. An empirical counterexample could falsify their theory (in Popper's sense).

However, compared to their overview of the Bénard problem, they were not able to give as detailed picture of the formation of the dissipative structure for chemical kinetics. "The only unified features at our disposal are the stability condition and the condition for the occurrence oscillations" (Glansdorff \& Prigogine, 1971, p. 222). The problem of the formation of chemical oscillations requests additional conceptual tools. Figuratively speaking, Glansdorff and Prigogine created the fourth level of thermodynamics, the level of mathematical theory of dynamic systems.

Glansdorff and Prigogine planned their chapter 'Time order in chemical reactions' in the following way:

We first consider the behaviour of oscillations on the thermodynamic branch [...] The situation is realized in the Lotka (1920) and Volterra (1931) model. We then study the oscillations belonging to a new non-thermodynamic branch. We discuss the difference with the behavior observed in the first case. The main feature is the advent of chemical oscillating process, the 'limit cycles' first introduced by Poincaré in the study of three-body problem (1892). It is very likely that these processes are of great importance for the occurrence of 'chemical clocks', which are typical examples of 'time-order' generated by dissipation (Glansdorff \& Prigogine, 1971, pp. 222-223).

Here Glansdorff and Prigogine refer first to the Lotka-Volterra kinetic scheme of an oscillatory reaction in the ecological context of the competition between prey and predator. However, this scheme leads to a set of periodic motions, each with a different period. As a result, it cannot be used to model chemical reactions with a well-defined period determined by the values of characteristic parameters such as rate constants and temperature.

Then they refer to the 'chemical clock'. The experimental basis of this discussion is the Belousov-Zhabotinsky reaction, the history of which has been described in the philosophy of science (see Pechenkin, 2004). Already Zhabotinsky and his coauthors applied the mathematical technique of Poincarés limit cycle to describe and explain this reaction. Prigogine and his coauthors worked in the 
same direction. In 1968, Prigogine and Lefever put forward the trimolecular model (the Brusselator), which mathematical characteristics are close to the mathematical properties that Zhabotinsky and his coauthors studied by discussing the mechanism of Belousov-Zhabotinsky reaction.

In Glansdorff-Prigogine's book, the Brusselator has not been mentioned. However, in Prigogine's (and his coauthors') subsequent books this model plays an important part.

So, leaving aside some hydrodynamic models, one can state that Glansdorff and Prigogine discussed two stable dissipative structures (Bénard cells and chemical clock-the Belousov-Zhabotinsky reaction) in their book on non-linear thermodynamics. However, the chemical clock cannot be understood within the framework of thermodynamics only. Here the theory of dynamic systems is essential.

This should be taken into account while the generalization of the concept of dissipative structure is taken under consideration.

\section{The extrapolation of the concept of dissipative structure}

In Glansdorff-Prigogine's book (with the exception of the concluding philosophical Chapter XVII), there is no extrapolation of the concept of dissipative structure. Glansdorff and Prigogine only write that their emphasis on chemical oscillations has been stimulated by a possible application of the elaborated concepts to biological problems. In Chapter XVII there is an extrapolation over biological structures (Glansdorff \& Prigogine, 1971, pp. 290-291), but it is emphasized that this extrapolation is tentative, presumable. Glansdorff and Prigogine (1971, p. 298) write that "it is certainly tempting to describe biological structure as open chemical systems operating beyond stability of thermodynamic branch. Such models applied to living systems are clearly incomplete, since much more information on the type of chemical reaction involved, would be necessary to explain essential features of life such as replication phenomena".

In Prigogine's book coauthored with Nicolis (1977), the list of dissipative structures has been expanded. The authors, however, warn that some of their "dissipative structures" are hypothetical. "The purpose of the present chapter is to give a general account of various models giving rise to dissipative structures and 
related phenomena. [...] We insist primarily on the variety and the wide range of applicability of the concept [...] Thus, certain parts of the present chapter have the character of review more than the comprehensive analysis." (Nicolis \& Prigogine, 1977, p. 160)

Here emerges another problem. The concept of dissipative structures is a thermodynamic concept. Nicolis-Prigogine's theory, as it is presented in their book, is mainly based on the theory of dynamical systems. The authors give warning that they could not reach a consistent presentation of their results on the basis of thermodynamics.

In the textbook by Kondepudi and Prigogine (1988) there is an extrapolation of the dissipative structures over Alan Turing's analysis of the mechanism producing the morphology of living organism. "We will briefly describe a Turing structure, or stationary spatial dissipative structure" (Kondepudi \& Prigogine, 1988, p. 445).

In the semi-popular book mentioned above, Prigogine (1980), by formulating the concept of dissipative structure, refers to a number of biological phenomena. First of all, he refers to glycolysis, the chain of metabolic reactions during which glucose is broken down and an energy-rich substance ATP (adenosine triphosphate) is synthesized providing an essential source of energy common to all living cells. For each glucose molecule that is broken down, two molecules of ADP (adenosine diphosphate) are transformed into two molecules of ATP.

Then Prigogine (1980, p. 123) writes that "other examples of oscillating feedback-producing mechanism can be found in the aggregative in slime molds, in reaction involving membrane-bound enzymes, and so forth".

Prigogine is careful in his extrapolations. "It is [...] very tempting to suggest that the origin of life may be relevant to successive instabilities somewhat analogous to successive bifurcations that led to a state of matter of increasing coherence" (Prigogine, 1980, p. 123) (italics by the present author).

This carefulness is absent in Prigogine's book coauthored with Stengers (Prigogine $\&$ Stengers, 1984). True, the authors write about self-organization rather than about the dissipative structures.

The situation around the concept of dissipative structure is described by a specialist in molecular biology. "It is becoming a good pitch to supply the concept of dissipative structures with examples from biology. Typically, these examples do not have a real theoretical base" (Belintsev, 1983, p. 17). 
The above quotation does not mean that Belintsev, whose paper has been quoted, is opposed to the concept of dissipative structure. However, he emphasized that every extrapolation of this concept over biological phenomena should be supported by serious theoretical research.

\section{From dissipative structures to self-organization}

Already in Prigogine's (1980) semi-popular book, there is a tendency to use the concept of self-organization instead of the concept of dissipative structures. It is understandable: behind the dissipative structure persists a rather rigorous analysis of such phenomena as the Bénard cells and chemical clock. The concept of selforganization is a descriptive concept which has no explication in the terminology of physics. As was said at the beginning of the present article, "self-organization is a process where some form of global order or coordination arises out of the local interactions between the components of an initially disordered systems".

In Prigogine's abovementioned book, Chapter 4, where the concept of dissipative structure is formulated, is subsequently followed by Chapter 5 titled "Selforganization". It is interesting that the term 'self-organization' is not present in this chapter. This is a chapter about stability of dissipative structures, the Brusselator, coherent structures in physics, chemistry, biology, ecology. The term 'selforganization' helps the author to group together many different topics. At the end of the book Prigogine cites Ramon Margalef's book on ecology, viewed in a very wide sense of the word. Margalef writes about the "baroque of the natural world".

What he means is that ecosystems contain many more species than would be "necessary" if biological efficiency alone were an organizing principle. This "overcreativity" of nature emerges naturally from the type of description being suggested here, in which "mutations" and "innovations" occur stochastically and are integrated into the system by the deterministic relations prevailing at the moment. (Prigogine, 1980, pp. 129-130)

In the work by Prigogine and Stengers (1984), 'self-organization' is becoming to be its ideological principle. This is expressed in the 'Preface' written by the writer and philosopher Alvin Toffler.

What makes the Prigogine paradigm especially interesting is that it shifts attention to aspects of reality that characterize today's accelerating social 
change: disorder, instability, diversity, disequilibrium, nonlinear relationship (in which small inputs can trigger massive consequences) and temporality-a heightened sensitivity to the flows of time.

The work of Ilya Prigogine and his colleagues in the so-called 'Brussels school' may well represent the next revolution in science as it enters into a new dialogue not merely with nature, but with society itself.[...]

In Prigoginian terms, all systems contain subsystems which are continually 'fluctuating'. At times, a single fluctuation or a combination of them may become so powerful, as a result of positive feedback, that it shatters the preexisting organization. At this revolutionary moment [...] it is inherently impossible to determine in advance which direction change will take: whether the system will disintegrate into 'chaos' or leap to a new, more differentiated, higher level of order or organization, which they call a 'dissipative structure'.

One of the key controversies surrounding this concept has to do with Prigogine's insistence that order and organization can actually arise 'spontaneously' out of disorder and chaos through the process of 'selforganization.' (Prigogine \& Stengers, 1984, p. xv)

Let us turn to the epilogue to the Russian translation of Prigogine-Stengers' book. This epilogue also emphasizes the concept of self-organization, but it puts an emphasis on the self-organization of the process of thinking. The authors write,

Order from Chaos assumes a personal, dialogue way of thinking - thinking as a process open for future, the irreversible communicative process developing in time. Such a dialogue is art which can't be entirely described by means of formal logic. In this dialogue there are no prepared answers to the asked questions and there is no final list of questions either. Each of the parties involved in such a dialogue is not only asking and it is not only answering. (Prigogine \& Stengers, 1986, p. 411)

Another reflection on Prigogine's writings can be found in Vyatcheslav Stepin's book Theoretical Knowledge (Stepin, 2005, pp. 346-347):

Further development of physics led to understanding of scantiness of idealization of closed systems and description of real physical processes in terms of such systems. The overwhelming majority of natural objects are open systems, which exchange energy, matter and information with the 
surrounding world, and a decisive role in the radically changed world passes to unstable, non-equilibrium states. Fundamental sciences dealing with nonliving nature-physics, chemistry, cosmology—more and more often faced the necessity to take these features into account. But the old theory turned out unfit for their description. The traditional paradigm could not cope with the growing multitude of anomalies and contradictions, leaving many discovered phenomena unexplained. There appeared a need to develop a fundamentally new approach, adequate to objects and processes drawn into the orbit of investigation.

An important contribution to such approach was made by I. Prigogine's school. Researches of that school demonstrated that, moving away from equilibrium, thermodynamic systems get fundamentally new properties and start submitting to special laws. At considerable deviation from equilibrium thermodynamic situation appears a special type of dynamic state of the matter-dissipative structures. According to Prigogine, the type of dissipative structure depends to a large extent on conditions of its formation, and external fields may play a special role in selection of the mechanism of self-organization.

This is a conclusion with far consequences, if we take into account its applicability to all open systems which have an irreversible character. Irreversibility is what is characteristic for modern non-equilibrium states. They "carry the arrow of time" and are source of order, engendering high levels of organization. (Stepin, 2005, pp. 346-347)

Stepin explains what the dissipative structure is by inviting the concept of self-organization. By forgiving him such a jump, one may conclude that his excursion into Prigogine's thermodynamics is typical for philosophical writings on self-organization. These writings take self-organization as a key concept which helps us to understand the world. It refers to the 'arrow of time' and express the development of a wide variety of systems toward higher levels of organization. In his other writings, Stepin (1992, p. 10) connects the concept of self-organization with the concept of post-nonclassical science expressing a new scientific revolution. The post-nonclassical science is represented by the theories which consider self-organization as a key concept. According to Stepin, this is nonlinear thermodynamics, synergetics, the general theory of systems. 


\section{The Weltanschauung philosophy as a presumption of philosophical talks on self-organization}

It is natural that scientists venture into philosophical discussions in the introductions and conclusions which are present in their books and specially write popular essays containing philosophical excursions and generalizations. Certainly, such philosophical discussions help their self-consciousness, and by means of philosophical essays scientists express their understanding of their position in science and in the world in general. By employing philosophy it is easier for scientists to explain their contribution to science and culture to nonspecialists.

Still, how does this home-made philosophy relate to the professional philosophy of science?

People who consider themselves philosophers of science often construct their philosophical positions on the basis of such philosophical excursions. They treat them as a kind of empirical material to construct their generalizations about the world, culture, and civilization. They also use them as a methodology of science and apply them to explain the general social and cultural shifts characterizing the contemporary life. They combine them with their professional conceptsparadigm, scientific revolution, research program, Weltbild (picture of the world), etc.

However, the philosophy of science constructed in such a way can not be useful as a methodology. Firstly, it is uncritical. It is not possible to critically analyze any conceptual scheme by being based on this conceptual scheme. Besides, methodology needs to clarify scientific knowledge and makes clear what looks obvious. The philosophy which is based on the scientists' philosophical talks takes for granted that level of clarity (and, correspondingly, roughness) which "now and here" has been reached by science.

Philosophical talks about self-organization are somewhat similar to what Edmund Husserl (1910) called Weltanschauung ('worldview') philosophy. "Weltanschauung philosophy presupposes all the practical science as treasures of objective truth, and insofar as it has its goal to satisfy as far as possible our need for thoroughgoing and unified all-embracing and all-penetrating knowledge, it looks on all particular science as its basis. In view of this, by the way, it calls itself scientific philosophy" (Husserl, [1910], p. 188). 
Husserl contrasts his project "philosophy as rigorous science" to what he calls Weltanschauung philosophy. Weltanschaunng philosophy uses "the admitted cliche" and "the now-beloved expressions". In contrast to the Weltanschauung philosophy, rigorous philosophy "through a classification of the problems and through penetration into their pure sense", develops the methods "adequate to these problems".

Within the present paper I was not interested in discussing the positive intention of Husserl's project. Critical intentions of Husserl's programmatic paper are important for us. This criticism made clear that philosophical analysis of scientific knowledge should not consist in constructing the "worldview" and, of course, it should not be reduced to a popular retelling of the popular retellings.

\section{References}

Belintsev, B. N. (1983), 'Dissipativnye struktury i problema biologicheskogo formoobrazovaniia' [Dissipative structures and the problem of biological pattern formation], Uspekhi Fizicheskikh Nauk, vol. 141, no. 1, pp. 56-101.

Chandrasekhar, S. (1961), Hydrodynamic and Hydromagnetic Stability, Oxford: Clarendon Press.

Feistel, R. \& Ebeling, W. (2011), Physics of Self-Organization and Evolution, Weinheim: John Wiley-VCH Verlag. http://dx.doi.org/10.1002/9783527636792

Glansdorff, P. \& Prigogine, I. (1971), Thermodynamic Theory of Structure, Stability and Fluctuations, London, New York, etc.: Wiley-Interscience.

Husserl, E. ([1910]), Philosophy as a Rigorous Science. Retrieved from https://www.scribd. com/doc/63651073/Husserl-Philosophy-as-a-Rigorous-Science-New-Translation, pp. 166-196 [accessed Oct 2015]

Kondepudi, D. \& Prigogine, I. (1988), Modern Thermodynamics: From Heat Engine to Dissipative Structures, New York: John Wiley.

Landau, L. D. \& Lifshitz, E. M. (1988), Teoreticheskaia fizika: Gidrodinamika [Theoretical Physics: Hydrodynamics], vol. 6, $3^{\text {rd }}$ ed., Moscow: Nauka.

Näpinen, L. (1993), 'Philosophical foundations of synergetic modeling,' Proceedings of the Estonian Academy of Sciences: Humanities and Social Sciences, vol. 432, no. 4, pp. 378-390.

(2004), 'Understanding of the world and the scientific paradigm of selforganization,' Studia Philosophica, Special Issue: Proceedings of the $7^{\text {th }}$ Summer Symposium of the International Society of the Philosophy of Chemistry, 16-20 August, Tartu, vol. 4, no. 40, pp. 146-177. 
(2014), 'A philosophical description of some features of the paradigm shift done by I. Prigogine,' Historiae Scientiarum Baltica: Abstracts of the XXVI International Baltic Conference on the History of Science, Helsinki, 2014, p. 36.

Nicolis, G. (1970), 'Thermodynamic theory of stability, structure and fluctuations,' IUPAC. Retrieved from http://pac.iupac.org/publications/pac/pdf/1970/ pdf/2203x0379.pdf [accessed Oct 2015]

Nicolis, G. \& Prigogine, I. (1977), Self-Organization in Nonequilibrium Systems, New York: J. Wiley.

Pechenkin, A. (2004), 'Understanding the history of the Belousov-Zhabotinsky reaction,' Studia Philosophica Special Issue: Proceedings of the $7^{\text {th }}$ Summer Symposium of the International Society of the Philosophy of Chemistry, 16-20 August, Tartu, vol. 4, no. 40, pp. 106-130.

Prigogine, I. (1977), Time, Structure, and Fluctuations. Nobel Lecture, December 8. Retrieved from http://www.nobelprize.org/nobel_prizes/chemistry/laureates/1977/ prigogine-lecture.html [accessed Oct 2015]

(1980), From Being to Becoming: Time and Complexity in the Physical Sciences, San Francisco: Freeman \& Co.

Prigogine, I. \& Stengers, I. (1984), Order out of Chaos: Man's New Dialogue with Nature, Foreword by A. Toffler, London: Heinemann.

(1986), Poriadok iz khaosa. Novyi dialog cheloveka s prirodoi [Order out of chaos. Man's new dialogue with nature], Moscow: Progress.

Robinson, R. (1954), Definition, Oxford: Clarendon Press.

Rumer, Yu. B. \& Ryvkin, M. Sh. (1980), Thermodynamics, Statistical Physics, and Kinetics, Moscow: MIR Publishers.

Stepin, V. S. (1992), 'Stanovlenie idealov i norm postneklassicheskoi nauki' [The formation of ideals and norms of post-nonclassical science], in Problemy metodologii postneklassicheskoi nauki, Moscow: Institut Filosofii, pp. 10-48. http://dx.doi.org/10.1007/1-4020-3046-0

— (2005), Theoretical Knowledge, Dordrecht: Springer.

Wikipedia (n.d.), 'Self-Organization.' Retrieved from https://en.wikipedia.org/wiki/Selforganization [accessed Oct 2015]

Alexander Pechenkin graduated from the Mendeleev Institute of Chemistry and Technology (Faculty of Organic Substances) and the Lomonosov Moscow State University (Faculty of Mechanics and Mathematics). He received his PhD from the Institute of Philosophy of the Academy of Sciences in 1968. $\mathrm{He}$ is professor of the Moscow State University and lectures courses in the history and philosophy of science. He also works for the Institute for the History of Science and Technology of the Russian Academy of Sciences. 